\title{
Fleet and revenue management in car rental companies: a literature review and an integrated conceptual framework
}

\author{
Beatriz Brito Oliveira* ${ }^{\dagger}$, Maria Antónia Carravilla*, José Fernando Oliveira* \\ * INESC TEC and Faculty of Engineering, University of Porto \\ $\dagger$ Corresponding author: beatriz.oliveira@fe.up.pt
}

\begin{abstract}
This paper aims to present, define and structure the car rental fleet management problem, which includes operational fleet management issues and problems traditionally studied under the revenue management framework. The car rental business has challenging and distinctive characteristics, which are mainly related with fleet and decision-making flexibility, and that render this problem relevant for academic research and practical applications. Three main contributions are presented: an in-depth literature review and discussion on car rental fleet and revenue management issues, a novel integrating conceptual framework for this problem, and the identification of research directions for the future development of the field.
\end{abstract}

Keywords: car rental; fleet management; revenue management.

\section{Introduction}

This paper aims to present, define and structure the fleet management research focused on the problems faced by car rental companies. The focus on the car rental context arises from the interesting and challenging idiosyncrasies of its fleet and decision-making processes, which have some structural differences when compared to other transportation sectors more traditionally studied in the literature. Moreover, car rental is a growing business, comprising $\$ 27.11 \mathrm{bn}$ in revenue in 2015 in the U.S. - which represented a $4 \%$ improvement over the previous year - while the average car rental fleet grew $5 \%$ (Auto Rental News, 2015). This growth trajectory has been steady since 2010 and is forecasted to continue. From 2016 to 2021, the global car rental industry is expected to grow 5.6\% (CAGR), due to increasing tourism activities, the globalization of operations, and the global rise of income levels (ReportsnReports, 2015).

The car rental fleet management problem embeds decisions that are traditionally framed within different strategic levels and studied by different research areas. The main decisions are related with clustering locations that will share the same fleet, deciding on the fleet size and composition, distributing fleet amongst rental stations, deciding on prices, selecting which reservations to accept, and assigning these reservations to specific vehicles. In a real-world setting, these decisions are not only linked by close interactions but also by overlapping decision-making time horizons. In fact, one of the main characteristics of the car rental fleet that motivates this study is its inherent flexibility. On the one hand, the fleet is significantly easy to move and re-locate, enabling e.g. the use of strategic fleet balancing decisions often referred to as "empty transfers". On the other hand, there is also a flexibility on the decision-making process that often renders the traditional hierarchical overview of fleet decisions too rigid. For example, the acquisition and removal of cars to and from the fleet is significantly flexible, as these contracts are often incentivized with small lead times, and frequently throughout the year.

Due to the relatively small number of papers that deal with this problem so far, this review aims to be exhaustive within its scope, which comprises quantitative methods that were developed to support decisions related with car rental fleet management. It is structured in three main parts. Firstly, the seminal works that launched the interest in the field are reviewed, which are generally accounts of the early implementation of fleet and revenue management systems in car rental companies. The second part is devoted to the main works, which have structured the field and set the ground for future works. These will be the focus of the third part, which is divided in smaller sections related with the type of decisions: the clustering of rental locations in groups that share the same fleet (pools) and the fleet management within each pool, which comprises operational decisions, revenue management decisions and the integration of both. 
Arising from the literature review, a conceptual framework is proposed to structure the car rental fleet management problem. The literature in the area is scarce and somewhat concentrated in only a few of the problems; however, the interest in this field has been growing in the past years and expanding to different sub-problems within this scope. The framework herein proposed aims to contextualize the relations between the different sub-problems, and is motivated by the need to support the development of methodologies that are applicable in real-world settings.

One of the main contributions of this work is also the proposal of four research directions. These are based on the framework and literature review and are related with the increase of the realism and applicability of the existing methods and the exploration of different levels of integration of the subproblems.

The remainder of this paper is thus structured as follows. In Section 2, the problem is introduced and described in detail; moreover, some "lessons learned" from research in other transportation sectors are discussed. Then, in Section 3, the literature review on the car rental field is presented and discussed. The framework for the problem is then presented in Section 4 and, based on it, the research gaps are identified. Four main research directions for the future are also proposed in this section. Finally, some conclusions are drawn and the main contributions and limitations of this work are discussed in Section 5 .

\section{Problem and contextualization}

In this section, the problem of fleet management in the car rental industry, which incorporates several interconnected sub-problems, is presented. The goal of this section is to informally describe and contextualize the main business decisions, with no specific intention to structure and thus limit the problem definition.

Fleet management is indeed a mature topic of research in other transportation fields. From some of these fields, such as the airline industry and maritime or rail-freight transportation, parallels can be drawn with the car rental business and thus useful lessons can be learned. Nevertheless, there are structural differences that support the need for a more specific treatment of the car rental business and these will also be presented.

\subsection{Fleet management in the car rental business}

The goal of this section is to broadly present the car rental fleet management problem and its main decisions. In fact, the car rental business profitability is heavily dependent on its fleet and all decisions that concern it. These fleet decisions span across all strategic levels of the company, from the network design decisions to specific-vehicle maintenance requirements. The following description focuses on the main decisions dealt with within this scope regarding the network design, the definition and utilization of the fleet, and the management of booking requests and consequent schedules for each vehicle.

Network In bigger car rental companies, the rental stations are usually aggregated in pools - groups of stations that share the same fleet. These pools are independent from other administrative divisions (e.g. regional divisions) although they can overlap; therefore, there is a certain flexibility to change and adjust them. In fact, this is not a "one-time decision" by nature; the pool design may be frequently reshuffled as a means to e.g. meet seasonal changes in demand patterns across locations. There is a specific set of cars assigned to each pool, to be shared by the rental stations that form it. Within the pool, the specific location of the car at a certain time depends on its status: if it is fulfilling a reservation, idle at a certain station, or under maintenance at a certain workshop. Ultimately, some cars may even be outside their pool, if a customer picks-up a car in a rental station that belongs to the pool but returns it to a station outside the pool.

Fleet definition A car rental fleet is composed of a number of cars of different types (rental groups). These groups may be substitutable, which will be discussed later when the reservation proceedings are described, thus connecting the decisions of "how many cars of a certain group to have?" for the different rental groups. Overall and generically, the size of the fleet is mostly determined by the company's strategic positioning and available investment, which is a one-time decision and out of the scope of the fleet management problem studied. Nevertheless, the operational adjustments made to increase and decrease the fleet, usually within a pool, are critical for a proper fleet management and will be herein analysed. 
A fleet management problem deeply linked, or even included, in deciding the size adjustments, is related with the vehicles to acquire and remove from the fleet. Actually, buying and selling vehicles can play a very relevant part on the company's profitability. This part of the process is extremely dependent on the type of car rental company. Some car rental companies are part of vertically integrated business groups, and thus have a close access to a manufacturer and/or to a wholesale reseller. For these companies, acquiring vehicles can be compared to a leasing contract, where a specific service deadline is defined for each car; they can also have access to discounted prices or other amenities. As for removing the cars from the fleet, they are more protected against fluctuations in the used car market, for example, as the responsibility to dispose of the stock falls on the reseller company of the group, or at least is shared with it. For the remainder of the companies, however, how the vehicles are acquired and removed from the fleet is as important as when. These decisions can also be significantly flexible, yet this depends on the mode of acquisition/removal. In fact, although some acquisition contracts must be made with some antecedence, the assignment of new cars to the respective pools can be made with a short notice, if needed. The decisions on the removal of cars from the fleet are also extremely important, especially since they can and should be made vehicle-by-vehicle. If the company wants to sell back the used car, specific information, such as the odometer values, is critical to decide on "sell dates" (Lacetera et al., 2011).

Fleet utilization Another critical decision is how to divide the existing fleet among the rental stations, within a pool. This is a critical aspect since the majority of the operational costs in car rental are related with idle fleet. That is to say, the ideal operational goal of car rental companies would be to have $100 \%$ of the fleet occupied $100 \%$ of the time. These decisions are extremely flexible, as the fleet levels at each station are constantly being changed due to incoming returns and pick-ups. Moreover, due to imbalances on demand and the possibility to rent here, return there, there may exist the need to empty reposition the vehicles between stations, either with a driver repositioning a specific car or by transferring a batch of vehicles by truck. These transfers, which are critical for balancing the fleet levels across the pool, or among pools, are extremely costly. These costs may be reduced with proper planning methods.

Booking requests and vehicle scheduling Fleet management in car rental companies also includes the task of assigning specific vehicles to booking requests. These requests can be made with some antecedence (reservations), enabling a pre-plan of this assignment, or by walk-in customers, which require a vehicle on the fly from a specific rental station. In some companies, this assignment is decided by the rental station staff. However, for other companies, this is planned in a somewhat centralized level (e.g. pool level), especially for rental groups which have a smaller number of cars available (e.g. luxury cars). Furthermore, it is also important to schedule the planned maintenance for the fleet vehicles. This can be done simultaneously with the scheduling of the reservations.

The main characteristics of a booking request are: the desired renting group, the pick-up (or checkout) date and station, and the return (or check-in) date and station. If there are no cars from the desired group available, it is common practice to offer an upgrade, i.e. a vehicle from a "better" rental group for the price of the originally requested group. Also, as a last resource to avoid a lost sale, some companies offer a possibility to downgrade, i.e. get a vehicle from a "worse" rental group for a discounted price. It is because of these strategies that it is critical for car rental companies to manage their fleet integrating all rental groups.

Due to the close links between demand and fleet occupation, and the importance that occupation has on the operational efficiency and cost structure of the company, the decision to accept or reject booking requests is also important when managing the fleet. Although some companies may fulfil all booking requests in order of arrival as long as there is capacity available, other companies manage demand by saving capacity for more profitable reservations that may arrive later, either by using complex segmentation, capacity allocation or pricing methods or simply by heuristically prioritizing reservations.

Uncertainty The fleet management problem in car rental is severely affected by uncertainty. Demand uncertainty is the issue most recognized and addressed by car rental companies, by in investing in accurate forecast methods, for example. Nevertheless, other factors bring uncertainty to the problem, with significant impact on fleet management. For example, when acquiring new vehicles and removing old vehicles from the fleet, the costs and profits associated with these decisions have a degree of uncertainty that can have a significant impact on the final decisions. Also, the availability of the fleet is often influenced by uncertainties such as unplanned vehicle maintenance and repairs or delayed car returns. 


\subsection{Lessons learned from other transportation sectors}

A myriad of sectors and industries have been using quantitative approaches to optimize or improve their fleet management processes. From maritime transportation to humanitarian aid, the need to efficiently manage a fleet of vehicles is extended across strategic levels and business functions. Academic research is more prominent in certain fields, namely in those where transportation is the core business; nevertheless, interesting and innovative applications have been rising. The process of retrieving the lessons learned from other sectors is often hindered by the lack of standardized problem names. For example, 'assignment' is a concept with several interpretations in terms of scope, inputs and outputs. 'Assignment' can either refer to allocating parts of the fleet to a specific location, to assigning a specific vehicle to a specific order/demand, or to assigning a type of vehicle to a type of order/demand. Despite these difficulties, in this section there is an attempt to understand what type of research has been developed in the fleet management context in different sectors, what parallels can be found with the car rental business, and what differences and challenges hinder its straightforward application on this field.

Airline industry The airline industry is a traditional "comparison sector" in the car rental literature. In terms of operational fleet decisions, there is indeed a strong body of research in this field regarding fleet, tail and crew assignment, amongst other issues. For a thorough review on assignment in the airline industry see Sherali et al. (2006). Moreover, as a pioneer in the field of revenue management, the airline industry is also often referred when dealing with capacity control decisions in car rental.

The focus on fleet management problems in the airline sector has been mainly motivated by practical issues the airlines have been faced with. For example, Salazar-González (2014) describe the analytical approaches used in a real application: a research project developed alongside a regional carrier, which worked on issues such as fleet assignment, as well as aicraft routing and other problems. These approaches were validated for real-world instances and are currently being employed by the company.

Fleet assignment in airline relates to assigning aircraft types with different capacities and different characteristics to the previously scheduled flights, based on availabilities, operational costs and potential revenues. This is different from the assignment of a specific physical aircraft to a flight, which is called tail assignment. Flight scheduling, on the other hand, is related with the flight network specification, including departure and arrival locations and times, working as an input to the assignment problem. Other critical fleet management issue in this field is the rotation problem, in which an individual aircraft can be assigned specific routes among those prescribed for its own type, while satisfying maintenance constraints. (Sherali et al., 2006; Clausen et al., 2010)

The tail assignment problem has strong similarities with the vehicle-reservation assignment problem in car rental, as the "services" for each specific physical vehicles are being scheduled. Also, the allocation of vehicle types to flight legs can be comparable with tactical decisions in car rental. In fact, time-space networks and the representation of connections and flight legs with arcs is often used in this field; this is also frequently considered in some car rental fleet decisions in Section 3. However, the main difference that motivates the specific study of the car rental problem is the mobility of the fleet, namely the possibility to empty reposition the vehicles. This flexibility comes from the costs that, although important, are still low enough to make these decisions profitable; in the airline industry, however, the costs to do the same are prohibitive. Moreover, in the car rental industry, the process of buying and selling vehicles comprises important decisions that, due to the business characteristics, turn out to be essentially operational, namely as far as timings and acquisition modes are concerned. In the airline industry, these variables are not usually considered alongside other fleet management issues and the decisions are taken in a more hierarchical and sequential way, once again due to the heavier costs associated with fleet decisions in this sector.

Maritime transportation Research on maritime transportation can bring interesting insights to car rental fleet management, especially regarding the decisions on size/mix. In this industry, the decisions on how many ships of each type are needed to meet demand are made periodically and often multi-stage approaches are used, thus representing the fleet renewal problem. Pantuso et al. (2014) present a thorough survey on these problems. The authors also state that models on fleet size often include the decision on assigning specific ships to pre-determined routes as well. In this industry, demand uncertainty is high, such as in car rental. However, the supply is much slower to adapt to peaks in demand as the lead time to acquire new ships is significantly higher than the one to acquire new cars. Nevertheless, one of the lessons learned from this industry should relate with the detail given to the mode of acquisition and disposal of the vehicles from the fleet. As examples, issues often considered are the possibility of chartering in and 
chartering out, or laying up, i.e. keeping the ship idle at a specific location with reduced crew and costs. Also in this field, it is critical to accurately represent fleet heterogeneity.

In summary, research developed on maritime transportation fleet management may bring significant insights for the car rental business, especially when the heterogeneous fleet renewal process is considered. Nevertheless, once again, the flexibility in buying and moving car rental fleet brings important advantages to the process and significant improvements may be gained by considering it explicitly (versus directly applying the research developed for maritime transportation to car rental).

Rail-freight In this context, the empty vehicle redistribution is core. Deciding the railcar distribution, i.e. where to send empty railcars to meet the next order, can be seen as an assignment to specific customers. Yet this is usually made at bulk, and not specifically for each vehicle. This problem has been studied since the 1990s (Spieckermann and Voß, 1995; Sherali and Suharko, 1998); nevertheless, it is still relevant and motivating research up to this date (Gorman et al., 2011). From these works, many parallels can be driven with the car rental business, as the mobility and flexibility of the vehicles is similar. One important idiosyncrasy of the car rental business, however, is that "orders" must be met without delay, at the risk of being lost, and cannot be, for example, backordered. In rail-freight, there is also plenty of research on fleet size, a problem commonly integrated with empty reposition decisions, such as in Sayarshad et al. (2010); nevertheless, this sector usually deals with a more homogeneous fleet than in car rental and substitutability between fleet types is not such a critical issue.

Trucking In the truckload carrying industry, several fleet management problems arise that have some parallels with the car rental industry. Powell (1991) reviewed optimization models and algorithms for problems such as the assignment of drivers to pending loads or the distribution of vehicles among locations and dynamically moving them to meet new demand. Several constraints make this problem significantly different from the car rental one, such as maximum tour length restrictions, time windows on pick-ups and deliveries, and the possibility to seize backhaul opportunities. Nevertheless, research in this area has been growing and, for example, fleet size and balance has been on the focus of recent works (Żak et al., 2011; Carbajal et al., 2012).

Shared-use vehicle services Shared-use vehicle services, such as car or bike sharing, are not a traditional transportation sector per se, since the academic and practical interest in this topic has emerged in the past decades. Nevertheless, since the mid-1980s, there is a growing interest in the academic community in this sector, related with different operational problems (Shaheen, 2013) that have also several similarities with the car rental fleet management problem. In fact, these similarities may allow for lessons from vehicle sharing systems to be learned by the car rental industry and vice versa.

Barth and Shaheen (2002) broadly define shared-use vehicle systems as fleets of vehicles used by different users throughout the day. The authors propose a framework for classifying these systems, which can follow different operational modes, such as car sharing, where a network of strategic parking locations is available for the user to pick-up and return the car, and station-car, where vehicles are deployed near metropolitan rail stations to be used by rail commuters. Gavalas et al. (2015) present a comprehensive review of algorithms for the management of shared-use vehicle systems, enabling a comparison of both sectors in terms of issues, goals, scope and approaches. The authors review the methods applied regarding the design of the vehicle sharing system - including network location, fleet size and deployment between stations -, which shows several similarities with the car rental business. The authors also focus on customer incentivisation schemes to help the balancing the fleet and on the operational improvement of the vehicles empty transfers.

In fact, key lessons can be learned from this sector especially regarding the deployment of the fleet between stations in order to meet unbalanced demand. Nevertheless, although the motivation is similar in both sectors, the business and operational approaches differ significantly (e.g. pricing schemes and vehicle reposition strategies). The main differences between the two sectors are related with the heterogeneity of the car rental fleet, and the "scale and scope" of the operations. The latter issue arises from the fact that vehicle-sharing systems have a daily focus, as defined by Barth and Shaheen (2002), and are usually centered in a single metropolitan area, while the car rental scope is usually wider in terms of time horizon and geography. These differences may lead, for example, to a greater need of detail (e.g. pricing in the car rental industry is usually almost individualized per reservation).

Nevertheless, the differences between the two sectors are becoming blurry as shared-use vehicle systems get operationally more complex (e.g. by introducing heterogeneous fleets). Therefore, besides the lessons 
learned, also some methodologies developed for the car rental industry may be adapted to meet future needs of shared-use vehicle systems.

Other sectors Other sectors deal with interesting fleet management problems, although research is not as developed as in the above-mentioned industries. For example, in underground mining, there is an interesting fleet management problem which involves dispatching load-haul-dump vehicles that move in forward or reverse mode on bi-directional segments of the network. In these systems, there are constraints on the capacity of the network, and so the continuous monitoring of the traffic on the network and reoptimization is critical. (Gamache et al., 2005; Beaulieu and Gamache, 2006)

In humanitarian aid, fleet management is also critical due to its impact on overhead costs. Martinez et al. (2011) focus on the importance of developing research in this specific field and, using a case-based approach, attempt to understand how this type of fleet is actually managed by international humanitarian organizations, what are the critical factors that affect it, and how it impacts the aid programs. Identifying optimal vehicle procurement policies, for example, is an important problem in this sector (Eftekhar et al., 2014).

Another interesting and different sector is presented by Perrier et al. (2007), who survey winter road maintenance research, including fleet sizing and fleet replacement models and algorithms for plowing and snow disposal. Also in fleet sizing, an interesting case study regarding the fleet of towing tractors in airports is presented by Du et al. (2015). The model developed in this work considers relevant realistic constraints such as vehicle lifetime allowed and different removal options, such as selling.

There are traditional sectors where fleet management optimization models and algorithms have been developed with detail for the past years, such as the airline industry or maritime transportation. From this body of research, the car rental sector can and has been getting insights and its basilar foundations; nevertheless, there are business specificities that motivate specific research in this field.

Therefore, in the car rental industry, although many lessons can be learned from traditional transportation sectors, there is also the need to develop quantitative methods for the fleet management problem that take in consideration the business specificities, thus enabling its actual use in the real-world context.

\subsection{Overview on the discussed problems}

In Section 2.1, the fleet management problem was described for car rental companies. Several subproblems were identified:

- The network division in pools of stations,

- The fleet size and the mix between different types of vehicles,

- How and when to acquire new vehicles for the fleet and how, when and which vehicles to remove,

- The distribution of the pool fleet among rental stations and how to make the vehicles available at the corresponding rental station,

- At what price to sell a specific product (combination of vehicle type, start date and location, end day and location, antecedence of the request),

- Which booking requests to accept and reject,

- What type of vehicle to supply for each accepted booking request,

- What booking requests to schedule to a specific vehicle, considering its specific requirements and attributes (e.g. maintenance requirements, planned removal date).

Some of the problems wherein considered are often studied under the revenue management framework, namely the ones where pricing and capacity allocation decisions are concerned. In the next sections, we will be arguing that fleet and revenue management issues should be integrated, due to two main reasons. On the one hand, there is often an ambiguous boundary between the two fields: for example, some authors defend that some decisions "traditionally" seen as more operational (e.g. deciding the number of vehicles) are actually functions of revenue management, and early records of the implementation of revenue management systems in car rental companies also included this type of operational decisions. On the other hand, even if one is able to define a clear boundary between the two fields, there are strong links between decisions belonging to different domains. In car rental, it is possible to observe that the main drivers of revenue are the demand, which is highly price-sensitive, and the occupation levels, which have a strong impact on the allocation of operational costs. For example, decisions on prices for different rental stations have significant impact on the demand levels in each station, leading to a need to re-balance the 
fleet levels in the pool in order to meet demand. In a different perspective, a specific pricing strategy can also be used in order to "push" demand for the rental stations where availability is higher.

It is also important to consider that these connections between "revenue management issues" and "operational/fleet management issues" are present in other industries as well. For example, Guerriero et al. (2012) address the problem of accepting/rejecting requests for a fleet of trucks of a logistics operator, which is seen as a capacity control revenue management problem, considering as well the operational/fleet management problem of empty repositioning trucks between locations.

\section{Literature review on car rental fleet management}

Literature on car rental fleet and revenue management is not plentiful, yet it has been blooming in the past decade. This literature review aims to present the different research streams in this field and discuss its main developments and opportunities. It is structured as follows. As an introductory note, the main contributions of the review presented in this paper are presented, comparing with the only review previously published for car rental fleet management. Then, the seminal works in the area, from the 1970s-90s, are presented. These describe the first experiments of car rental companies with revenue/yield management as well as fleet management decisions, following the ones in the airline industry. Afterwards, two basilar works from the 2000s that made significant contributions, either by structuring the field or by adapting the general models to the reality of the business, are discussed. The remainder of the works on the field are then presented, organized by problem/issue. To the best of our knowledge, the 23 papers analysed within the next section comprise the full body of work to date on car rental fleet management. Figure 1 represents graphically the structure of the proposed literature review and will be used as a pointer in the remainder of this section.

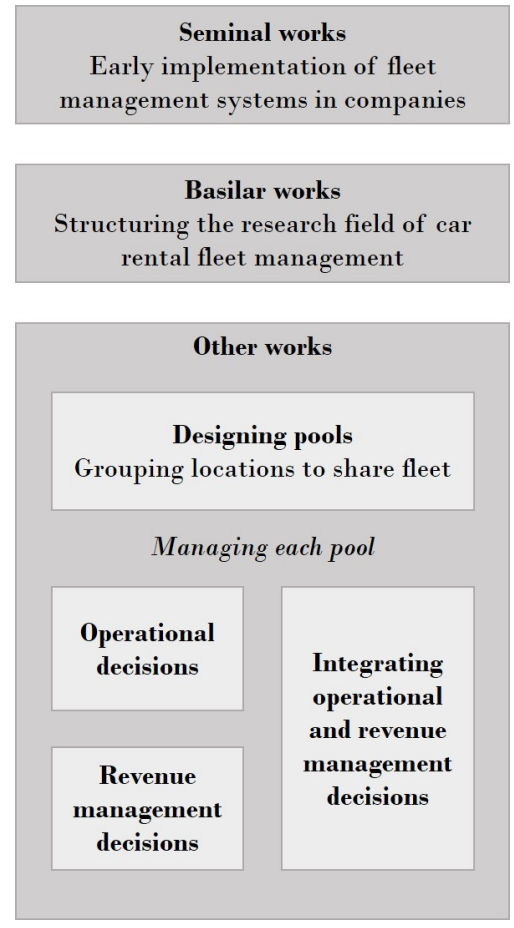

Figure 1: Literature review structure. This figure represents the structure of this section; the blocks represent the type of papers reviewed, which are divided in three main categories. The last category is divided in smaller sub-categories, by sub-problem.

A discussion is then presented, based on revenue management and operational issues in the car rental context, with a small note on the general size of the problems. The approaches and methods used for the different problems are also reviewed. 


\subsection{Literature review}

Yang et al. (2008) propose a review of the literature on the car rental logistic problem. Due to the scarcity of literature on this topic to date, the authors compare some specific problems with the ones faced by the airline industry. In fact, similar problems are relevant in both industries. However, there are significant idiosyncrasies of the car rental business that justify a more detailed analysis of the sector and there is sufficient potential of growth in this area that justifies a more challenging/critical approach to the proposed frameworks. The review by Yang et al. is descriptive and heavily dependent on the work of Pachon et al. (2006). Nevertheless, it suggests some interesting future research directions, such as the focus on vehicle-reservation assignment, which would later be developed (Hertz et al., 2009; Oliveira et al., 2014). It also pinpoints the importance of better demand forecast models, which include more realistic features such as no-shows; it will be argued in this chapter, however, that the need to include uncertainty should be considered in a broader manner than forecast models.

Thus, a more recent, exhaustive, critical and ground-building review was in need, which could enable a reviewed and comprehensive framework of the car rental fleet management problem.

\section{Seminal works: arising from the airline industry}

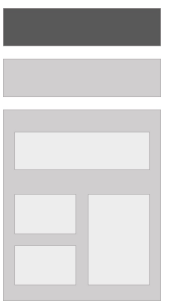

Research in the field of car rental fleet and revenue management arose from the industry and the first accounts describe the implementation of decision-aid systems in main car rental companies. Interestingly, since early on, the boundary between fleet management and revenue management often appears as somewhat blurry and the same decisions are often considered under different frameworks.

The first academic work in the car rental setting was published in 1977 and presented a decision support system (DSS) developed for pool control in Hertz Rent-a-Car. The implementation and analytical models developed are presented, involving strategic and tactical decisions, such as pool design, fleet size, and fleet deployment. (Edelstein and Melnyk, 1977)

The implementation of a yield management DSS also in Hertz Rent-a-Car is described in Carroll and Grimes (1995). Four main questions are answered by this system: "how many cars should Hertz have?", "where should it deploy its cars?" (fleet management), "what products should it offer?", and "what products should it sell?" (revenue management). Some important lessons can be derived from this early yet realistic and applied work. Regarding fleet size, the authors confirm the importance of the relationships with manufacturers and resellers, when planning acquisitions and removals. In fact, the structure of manufacturers' purchase plans and the means to dispose of used cars through retail car sales or through wholesale markets are pinpointed as complicating factors of the problem. The authors also make an important note regarding the two levels or perspectives of fleet size. It is important to distinguish between the strategic, overall definition of fleet size (long-term), and the adjustments made (either long or short-term), in which the system is focused. The revenue management focus is present in the two last questions, where product segmentation and capacity control mechanisms are implemented.

In the same decade, National Car Rental is also reported to achieve significant gains with the implementation of a revenue management system (Geraghty and Johnson, 1997). This system also controls the fleet planning process, such as empty transfers between stations, accelerating or retarding returns of vehicles, and redirecting new cars for the rental locations. This paper is somewhat more detailed in the methods used. It includes upgrading and overbooking decisions, as well as an heuristic to set prices based on an elasticity model that relates historic rate and demand variability.

Later, also Dollar Thrifty Automotive Group described their efforts on Revenue Management, namely their efforts on measuring the impact of these decisions using the 'Performance Monitor' system. (Blair and Anderson, 2002; Anderson and Blair, 2004)

As a conclusion, it can be observed that the first academic works concerning car rental fleet management were generally focused on describing the practical implementation of decision-aiding quantitative methods and tools in car rental companies, as part of complex decision support systems that deal with several issues of fleet and revenue management. Therefore, this is a field that was born from a practical need in the industry, where most relevant problems and issues were generally tackled within an integrated system. 


\section{Basilar works: structuring the field}

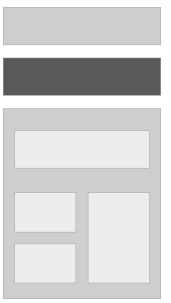

The basilar modelling framework for the car rental fleet management problem is set in Pachon et al. (2006). The main contribution of this paper is a sequential and hierarchical structure, which divided the planning process in pool segmentation - clustering rental locations in pools that share the same fleet -, strategic fleet planning - deciding fleet size for each pool -, and tactical fleet planning - deciding fleet levels for each location within a pool, and consequent "empty transfers" between locations. Besides the modelling framework, the authors propose solution methods to deal with its computational burden. Although presenting some oversimplifying assumptions, the formulations presented were the cornerstone for future works. In the strategic fleet planning step, when deciding the optimal fleet size, the authors also decide on the number of acquisitions and removals, although only in the form of leasing from and returning to the manufacturer. Also, substitution between vehicle types is not considered and hence the problems are separable by car type.

The authors consider that determining the optimal fleet size and mix for each location on a daily basis is a "primary function of revenue management". This supports the claim for a comprehensive overview of these problems and leads future works towards this goal, by considering the integration of "traditional" revenue management functions of pricing and capacity control in the fleet management framework. The operational problem of assigning accepted booking requests to specific vehicles, which is not considered in this framework, is also a clear example of ambiguity between fleet and revenue management in car rental, since it links operational issues, such as empty repositioning, and revenue management issues, such as capacity control.

Other core work in the car rental fleet management area is developed by Fink and Reiners (2006) that propose a realistic approach to the fleet size and mix problem, considering acquisitions and removals. This paper presents a model for this problem that includes several real-world issues that make this a realistically implementable model, such as considering multi-periods, a country-wide network, groups with partial substitutability, among other characteristics. Other contributions include a detailed description of the problem faced by car rental companies, with key details such as the typical life cycle of a car. Also, the authors propose a system architecture for a DSS that includes the optimization model. A relevant simplifying assumption in this work is that its scope excludes the relationship with car manufacturers and resellers. Therefore, the acquisitions and removals are, as before, seen as leasing contracts with virtual depots for car pickup and return, not accurately representing the actual buy-and-sell process.

\section{Other relevant works: developing the field}

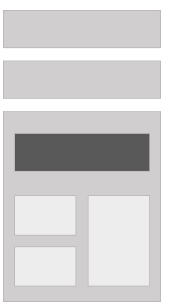

Designing fleet-sharing pools Following the framework proposed by Pachon et al. (2006) and starting with the arguably most long-term and strategic decision - pool segmentation -, it is possible to conclude that it has not received much attention from the research community. Yang et al. (2009) study the problem of grouping locations in pools with the objective of minimizing the number of pools with a similar approach to the one proposed by Pachon et al. (2006), yet they also consider the decision on the pool logistic center, i.e. the rental station that will be coordinating the shared fleet. The authors propose a model and an approximation algorithm. One key issue pointed out by the authors is that defining the pool should encompass some flexibility, as this design is not directly correlated with administrative delimitations. This supports the claim that capacity decisions can easily be reviewed periodically, even on the design of the pools of locations that share resources.

\section{Managing the fleet in each pool}

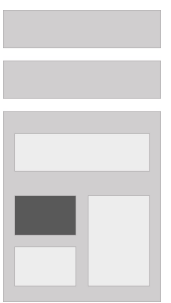

Focus on fleet/operational decisions Within each pool, the decisions most often considered are the fleet size - how many vehicles to have in a specific pool - and deployment how to distribute the fleet among locations, and how to empty reposition the fleet to achieve that. In fact, there are two perspectives in the literature regarding fleet size, defending that it should be set either i) considering each pool independently or ii) all pools simultaneously, at the time of pool design. Perspective ii) is presented in Pachon et al. (2006) while perspective i) is adopted by the remaining papers that deal with fleet size, where all rental locations are considered as part of one inseparable pool. The problems of fleet size and fleet deployment, typically with different decision time horizons, are often solved in an integrated manner. You and Hsieh (2014) model these two problems with a mixed-integer non-linear formulation and proposed a hybrid 
genetic-based algorithm to solve it. The main limitation of this work is the oversimplifying assumptions made. For example, the authors consider that all rentals take only one day, and thus, at the end of the day, the cars are all returned to a certain station.

Also Li and Tao (2010) deals with both problems, presenting a two-stage dynamic programming model where the fleet size is the first-stage decision and the vehicle transfer policy is the second-stage decision, as well as an heuristic approximation that shows good performance in determining fleet size. This work assumes that there are no lost sales, as it is possible to subcontract capacity. Other assumptions, however, can be challenged for their realism, namely that there are only two rental stations and that all rentals last only one day.

Song and Earl (2008) propose an event-driven model, not specific for car rental, that integrates also fleet size and transfer. The authors show that the policy for empty repositioning is of threshold control type; the explicit form of the cost function under threshold control is derived and used to calculate optimal fleet size and threshold values. Uncertainty in empty vehicle repositioning time is modelled using an exponential distribution yet it is shown that the method carries over to a range of distributions. No lost sales are assumed and an extension for hub-and-spoke systems is presented. Nevertheless, as the focus is not solely in car rental, some assumptions may not be completely adjustable, namely considering the system as only "two-depot" and considering that the arrival of loaded vehicles (which is, in the car rental context, the check-in of a reservation) is determined only by travel time.

Pachon et al. (2003) study the fleet deployment problem for car rental companies, considering fleet size as a given parameter. A stochastic model representing the problem is proposed, and then decomposed into two-sub-problems: deployment - decide fleet levels in each station -, and transportation - decide how to reposition cars among stations. The deployment sub-problem is formulated and solved as a static inventory control problem and the transportation sub-problem as a linear optimization program. A heuristic is developed to reduce the gap of the decomposition approximation. Some extensions are also considered: the cost of unsatisfied demand and excess fleet, service level constraints, and price elasticity of demand, where the authors present the sufficient conditions of optimality and then retrieve from the literature a price-elasticity demand function that fulfils them. Despite considering only one type of car and one-day rentals, the models proposed are still today significantly relevant.

The operational decision of assigning vehicles to reservations is usually studied as an isolated problem. Ernst et al. (2011) present a mathematical formulation for the assignment problem and its Lagragean dual problem. To solve this formulation, the authors use the Wedelin method by incrementally updating the Lagrangean multipliers. They also propose an heuristic based on the upper and lower bounds found, that shows a good performance on building the schedules and also on providing good lower bounds. This model considers multiple types of vehicles with substitution, planned maintenance requirements and planned vehicle disposals for specific vehicles. The schedules are meant to be rebuilt daily, although protecting already accepted reservations.

In Oliveira et al. (2014), a network-flow model formulation of this problem is presented, considering interdependencies between rental groups, vehicle maintenance and disposal, and also different reservation priorities. The authors propose a relax-and-fix heuristic procedure, which includes a constraint based on local branching that enables and controls modifications between iterations.

Hertz et al. (2009) solve the assignment problem in car rentals assuming that each day it is possible to buy and/or to subcontract more cars to satisfy the requests and also considering that some maintenance hours had to be scheduled for each vehicle within certain constraints. The authors propose an heuristic solution that combines two tabu search procedures with graph optimization techniques. The main difference is related with the capacity constraints; there is herein a tacit understanding that the fleet size is a tactical or short-term decision: if requests exceed the stock, it is possible not only to upgrade but also to subcontract or buy new cars. The constraints on maintenance are detailed and consist on the maximum time of use without maintenance and a capacity constraint on maintenance work, which is characterized by duration and number of workers. The main difference between this work and Oliveira et al. (2014) is that Hertz et al. (2009) do not consider that it is possible to reposition vehicles for demand to be fulfilled. Therefore, the formulation is focused on the dimension of "time" rather than "space": the reservations are not characterized by their starting and ending location, and the vehicle availability is analysed in terms of time (when it will be available), not considering the location where it will be available. This work arises from the ROADEF'99 international challenge, where these details were set. The authors also describe four other heuristic approaches presented in the challenge to the same problem, and compare the results obtained. 
Focus on revenue management decisions Research in these car rental issues has recently been blooming, namely under the revenue management framework, especially regarding capacity controls. That is to say, the problem of whether to accept or reject the booking requests that arrive. Conejero et al. (2014) actually tackle this problem without explicitly considering it a part of the "revenue management functions". The authors model this problem as a time-expanded network and propose an iterative algorithm to solve it. A first algorithm checks for admissibility (i.e. whether a reservation can be accepted) by finding a maximum flow on an auxiliary network, based on the Ford-Fulkerson approach; the authors then propose an iterative method based on a simplification of the auxiliary network. This paper is focused on the impact of one-way reservations in the fleet (im)balance. As the main application of this work is for the rental of electric cars, this is especially critical, due to the constraint on space for charging on drop-off. The main limitation of this work is the non-existence of empty repositioning flows. The aim of the work was indeed to balance the fleet without recurring to the repositioning; nevertheless, it would be interesting to analyse the profitability of its implementation.

Guerriero and Olivito (2014) study the issue of accepting or rejecting reservations using revenue management techniques. The authors propose a dynamic programming formulation and use linear approximations - i.e., static models solved "dynamically" by updating demand and capacity information - to derive acceptance policies based on booking limits and bid prices. The authors consider the existence of walk-in booking requests and the possibility of upgrading. The performance of both policies is compared under different circumstances.

The main focus of Steinhardt and Gönsch (2012) is the integration of the accept/reject decisions with planned upgrades. The authors propose a dynamic programming formulation, and two decomposition approaches (in days and in resources) and heuristics to solve the problem. This work also has a significant contribution to the utmost relevant discussion on the concepts, importance and implementation of upgrading mechanisms in car rental (see Section 3.2 below).

Regarding pricing decisions, Oliveira et al. (2015) describe the implementation of a DSS to update prices for a car rental company in the websites of e-brokers that compare prices in the market. The decision on price updates are controlled by an adaptive heuristic procedure, which is based on actual and desired occupation levels.

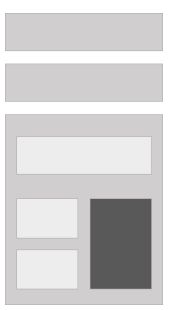

Integrating both perspectives In Haensel et al. (2012), the capacity control problem is integrated with fleet management decisions, more specifically fleet deployment and fleet repositioning. In this paper, a two-stage stochastic programming model for booking limits and transfer decisions for one type of car is proposed. The first-stage decisions are related with the capacity control (booking limits) and vehicle transfers and the second-stage decisions, after the uncertain demand is disclosed, represent the number of capacity actually "sold". A small case study is used to compare the deterministic and stochastic versions of the model. One simplification that can arguably cause significant changes in the structure of the problems is the fact that only round-trips, which start and end in the same rental station, are allowed.

To the best of our knowledge, only Madden and Russell (2012) deal with pricing decisions integrated with fleet management issues in the car rental context. In this work, the authors tackle the issue of pricing together with fleet deployment. In fact, it is interesting to investigate the similarities and differences between the two approaches - quantity-based and price-based revenue management - in the context of car rental (see Section 3.2). Madden and Russell (2012) propose an integer model based on a time-space network of rental locations, each with supply and demand for various car types based on the pricing level, that optimizes the choice of price levels together with relocation decisions. The dimensionality of the problem derives from the discrete approach to the choice of price levels and thus a linear programming formulation solved on a rolling horizon basis is proposed as an approximation. This unique formulation is based on the idea that pricing should help re-balance the fleet, through its impact on demand. Nevertheless, with this approach there is still the need to accurately describe the relationship between price levels and demand, a vulnerability which often makes the implementation impractical.

A different approach to revenue management, specifically for the car rental business is proposed by Anderson et al. (2004). The goal is to define acceptable prices and number of cars available for rent at a given price. The authors show that car rental is similar to "swing contracts" in electricity or gas markets, as the company is holder of swing-like options on car rentals. Prices are random variables, function of the remaining time to the start of rental and available inventory, modelled by a stochastic differential equation. In this work, only one type of vehicle is considered, and no upgrades are allowed. Some notes 
regarding the behaviour of prices are interesting to analyse, such as: although prices fluctuate, they seem to be bounded above, due to the "competitive, winner take all, nature of car rental market" and the price elasticity of consumers, and below, due to the marginal costs. As in other approaches, still, the slope of the demand curve is needed as a parameter.

\subsection{Discussion}

\section{Revenue management issues in car rental fleet management}

Based on the literature on car rental fleet and revenue management, it is possible to conclude that there is some degree of ambiguity between these two functions, derived from the many conceptual links that exist between the two types of decisions in a real-world setting. It is therefore important to clarify what types of decisions revenue management traditionally studies and critically assess how it has been applied in this sector, both in practice and in academia.

Van Ryzin and Talluri (2005) categorize revenue management as quantity-based if its primary tactical tool for managing demand is based on capacity-allocation decisions or price-based if it is based on prices. The choice between these two tools is dependent on the business context and on the flexibility the company has to change each of the variables, among other factors. Some industries traditionally use more quantity-based revenue management, such as airlines, while others use more price-based revenue management, such as retail.

Most previous research works on car rental focuses on capacity controls (quantity-based revenue management) (Conejero et al., 2014; Guerriero and Olivito, 2014; Steinhardt and Gönsch, 2012; Haensel et al., 2012). With the following section, we will support the claim that tackling pricing decisions is also important and adequate for car rental companies and has the potential to bring some added value to the discussion. First, the main logical reasoning to favour quantity-based revenue management will be de-constructed, building on general basilar works on both streams of research. Then, some relevant works that have been attempting to integrate or provide a common framework for the dichotomy quantity-price will be presented.

Overview on capacity allocation and pricing decisions Netessine and Shumsky (2002) introduce the field of yield management, focusing on capacity allocation decisions. The authors present the main motivation for firms to practice yield management and present the traditional tools for capacity control (booking limits, protection levels and overbooking) as well as other extensions. Herein, the authors discuss at a high level the main idiosyncrasy of car rental: the variation and mobility of capacity.

Also Van Ryzin and Talluri (2005) discuss the application of capacity control tools and their applicability to different sectors. Although not considering the specific case of car rentals, the discussion around airline companies and the reasons why they use capacity-controls may be of some interest. For airline companies, it is argued that "traditional" airlines (versus "low-cost" airlines) commit to prices on an aggregate origin-destination level and not on a departure-by-departure basis, which hinders the utilization of price-based revenue management tools. Moreover, the allocation of the resources to the different fares is extremely flexible, though subject to the capacity of the flight.

In fact, it is in the differences between these two business models that one may find the support for a different reasoning in car rental. As mentioned before, car rental companies are not subject to the same capacity constraints as airline companies, as it is easier to acquire, move and remove capacity. Moreover, car rental companies usually price their products not on an aggregate level, differentiating, for example, weekdays and weekends. Furthermore, even in the airline business, there have been changes in the past decade with the emergence of low-cost carriers and the proliferation of their pricing approach to the rest of the sector, causing a change of paradigm in practice. For example, nowadays it is easy to verify that even "traditional" airlines price on a departure-by-departure basis. Related with this, McAfee and te Velde (2006) present an interesting study that confronts the theories in the literature on airline dynamic pricing strategies with data depicting the companies' actual pricing behaviour.

Another reason that could support a hypothetical claim that quantity-based revenue management is the only adequate approach in car rental is that this is a highly competitive market and therefore companies are price-takers. Nevertheless, Talluri and Van Ryzin (2006) dedicate a chapter of their book to the relationship between economics and revenue management and claim that, although revenue management can be seen, at first, as a kind of anomaly from the classical economic models (for example, the wide dispersion of prices in the airline market may not be expected under intense competition), in the real-world contexts there are many economic forces at play that should be considered. For example the authors demonstrate that, even in a perfect competition setting, if there is a pre-commitment to capacity 
and demand is uncertain, price dispersion, either among companies or within the same company, is the unique competitive equilibrium; this is derived from the structure of the competitive market and not from the revenue maximization goal per se. For perfect competitive markets, this is also true when peak-loads exist, even if uncertain, or advance purchase discounts are applied. Other perfect competition, monopoly and oligopoly situations, possibly more adequate for the car rental business, are also analysed by the authors.

In fact, nowadays, pricing is getting more and more dynamic, since it is possible to gather data in real time and since the internet allows the price-updating process to be significantly easier and faster (Bitran and Caldentey, 2003). In this work, Bitran and Caldentey review the main pricing models in revenue management and their importance within the capacity and inventory decisions and claim that prices are very efficient variables that managers can use for controlling demand. Also, Şen (2013) shows that the use of dynamic pricing strategies may have a significant impact on the revenue of companies, even if simple dynamic heuristics are used to change prices based on the remaining product inventory. The author aims to emphasize the impact and benefits of this practice, which had been, on this perspective, not as present as needed in the revenue management literature, mainly due to the inherent computational difficulty of the method.

One may thus conclude that the logic and arguments that excluded pricing decisions from the demandcontrol toolbox of revenue management in this context have been vanishing with recent developments in the technology and business models used by car rental companies.

Integration of capacity allocation and pricing decisions To the best of our knowledge, the first seminal work that attempted to integrate pricing decisions with allocation decisions in a similar context was authored by Weatherford (1997). Here, different types of joint pricing and allocation problems for a perishable-asset problem are studied, considering either the presence and absence of demand diversion and nesting. With this approach, the prices, which were given as inputs for traditional models, are considered as decision variables alongside the capacity allocation.

More recently, Feng and Xiao (2006) study the integration of pricing and capacity allocation for perishable products with significant contributions, namely the notion of maximum concave envelope for an arbitrary set of prices. In this problem, at any time one or more customer classes are served and other classes may be declined. After choosing a class to serve, the pricing decision occurs, selecting from within a specific price set. Demand for each price is modelled as a continuous Poisson process and its intensity is dependent on time. Aiming to fill the capacity at the highest possible prices, the suppliers must decide simultaneously which classes to serve and at what prices. Despite the significant contributions to solving this problem, the authors recognize that the assumption on the ability to set prices might be too restricted. Also, a possible challenge of this integration is highlighted, as this perspective may invalidate the most favoured nested policy in capacity allocation, which defends that if a certain class is served then all higher classes must be served.

Following the efforts to integrate the two perspectives, Maglaras and Meissner (2006) propose a common formulation for a dynamic pricing strategy and a dynamic capacity allocation rule that controls when to accept or reject new requests for a multi-product situation. Another significant contribution from this work is a useful simplification for the multi-product dynamic setting: an equivalent formulation in terms of resource consumption rather than demand rates that significantly reduces the dimensionality of the problem.

Finally, some authors defend that, when given the choice between price-based or quantity-based revenue management - which has been most used in car rental -, it is possible to argue that pricing is the most advantageous approach, as it achieves the same function as quantity-based tools - rationing supply and limiting sales - but doing so in a more profitable way (Gallego and Van Ryzin, 1997). Nevertheless, the authors favour integrated approaches, defending that "there is a growing consensus among researchers and practitioners alike that the pricing decisions that induce demand cannot be separated from traditional, capacity-oriented yield management decisions; these two decision are inextricably linked".

It is thus possible to conclude that there are relevant arguments that support the utilization of price as a tool to control demand in car rental, especially if integrated with quantity-based approaches such as capacity allocation.

\section{Operational issues in car rental fleet management}

There are some key operational issues in car rental which are interesting to analyse due to their relevance in different works. Upgrading is a very important tool used in the car rental business and is often 
overlooked or oversimplified in academic works. The empty repositioning of vehicles is also extremely relevant for most of the fleet management decisions and is considered with different levels of detail in the literature. There is also a significant variance in the costs included in the objective functions, so the cost components will also be analysed in this section. A brief overview of the profit/value of rentals for more operational models (rather than revenue-oriented ones) is also presented. Then, a discussion is proposed on how the uncertainty that affects different processes is tackled. Finally, there is a small note for the disparity on the time-horizon assumed for different fleet and revenue management decisions in car rental. This discussion will be based on the basilar and later works presented before.

Upgrades Upgrading strategies are very common in the car rental business. They are built on the concept of substitution among different car types. When a car type requested by a customer is not available and a car of a "more desired type" is offered at the price of the original car, it is called an upgrade (Steinhardt and Gönsch, 2012). In this work, the authors discuss at length the upgrading strategies and their impact in car rental and state that the two main considerations are fairness and scope. The issue of fairness implies that upgrade priority is given to customers who purchase higher quality products. The scope is related with the extent of the substitution relationships between groups/products. The authors distinguish between and consider both full cascading - a group can be upgraded to any higher group (approach followed by (Pachon et al., 2006; Hertz et al., 2009)) - and limited cascading - upgrades are only allowed to the next higher group (considered by Conejero et al. (2014)).

On the one hand, the concept of fairness is often overlooked or dealt with only implicitly. On the other hand, the scope of the upgrade is often discussed and other intermediate extents are considered. For example, Guerriero and Olivito (2014) and Oliveira et al. (2014) consider that the allowed upgrades are mapped into a matrix, and in Fink and Reiners (2006) upgrades are allowed up to two higher groups. Some authors do not explicit the upgrading strategy followed yet mention that substitution is allowed (Madden and Russell, 2012; Ernst et al., 2011) There is also a lack of a common notation for the upgrading strategies: for example, Steinhardt and Gönsch (2012)'s "full cascading" is also labelled as "nested demand" in Pachon et al. (2006).

Steinhardt and Gönsch (2012) also discuss two different upgrade mechanisms: an ad hoc mechanism, where the firm must immediately decide to upgrade when an upgradeable product is sold, and a mechanism that postpones the decision until the customer picks up the car. The generality of works does not refer the choice between these two mechanisms, as it seems to be most dependent on the problem.

In fact, upgrades are critical not only for the business but also for the model formulation. If there is no substitution between car types, the model can be separated by type and the complexity is significantly decreased, which is a reason why some works consider only one car type and, consequently, no upgrades (You and Hsieh, 2014; Haensel et al., 2012; Li and Tao, 2010; Song and Earl, 2008). Nevertheless, due to their frequency in real-world settings, realistic models do consider, at whatever extent, upgrading strategies. The choice of this extent contains a trade-off in itself: although higher upgrade flexibility leads to a higher fleet utilization, in the long-term the customers might "learn" the strategy and start to require lower-valued groups leading to revenue degradation (Fink and Reiners, 2006).

Finally, other options considered to fulfil demand for unavailable car groups are downgrades, i.e. as a last resource, offering a car from a lower group at a lower price, in Oliveira et al. (2014), and sub-contracting capacity (Hertz et al., 2009).

Empty transfers Vehicle empty repositioning is a critical part of most fleet management problems in car rental. In the literature, however, this process is modelled following different representations, regarding both transportation time and mode. As for the duration of the transportation, some authors aim to approximate the actual transportation time, requiring that a matrix can be defined and given with the time the empty transfers take between all possible locations (Fink and Reiners, 2006; Guerriero and Olivito, 2014; Oliveira et al., 2014). This approach is more realistic although it can demand higher pre-processing difficulties when defining the time matrix. Other works assume that all vehicles can be transported overnight and simplify the modelling process (Pachon et al., 2006, 2003; You and Hsieh, 2014; $\mathrm{Li}$ and Tao, 2010). The main downside of this approach is not the capping assumption on the transfer time, which can be guaranteed by the pool design, but resides on the limitation imposed on the transferring schedules. For the vehicle-reservation problem, for example, it might have a significant impact if transfers between close stations are allowed during the day.

The empty transfers can also be materialized in different modes. Fink and Reiners (2006) distinguish transfers by truck, by driving the car itself, and using combined options such as driving the car up to a point, from where it is sent by truck to the final destination. Also Song and Earl (2008) consider different 
modes that are characterised by different speeds; yet this is applied on a context related with containers rather than cars. No other work that is focused specifically in the car rental business considers different types of transfer when tackling the problem.

Costs Most fleet management problems in the car rental context are formulated as cost minimization problems, and even in the ones formulated as revenue maximization problems the costs play a significant role, especially if they are realistically defined. Depending on the specific problem and the degree of detail of the models in the literature, different types of costs are considered. The list below presents the most critical ones found throughout the car rental fleet and revenue management literature; thus, each work generally considers a combination of these costs:

- Acquisition costs: usually considered per vehicle (Pachon et al., 2006; Hertz et al., 2009). Since the buy-and-sell relationships are out of the scope of most works, adding and removing cars from the fleet is often modelled as a leasing-type activity, not including acquisition costs, or including them in a "per vehicle basis", overlooking the economies of scale, contracts and other realistic characteristics;

- Holding costs:

* Leasing/sub-contracting costs, per unit of time (Pachon et al., 2006; Hertz et al., 2009; Song and Earl, 2008);

* Operating/stocking costs, per day (You and Hsieh, 2014; Hertz et al., 2009);

* Maintenance costs, per maintenance session and depending on type of car (Hertz et al., 2009), or per car and per day, depending on the current location (Song and Earl, 2008);

* Penalty per day of delay in returning the car, if it is leased (Fink and Reiners, 2006; Pachon et al., 2006).

- Empty transfer costs (following from the discussion above):

* Transfer cost per car, depending or not on the origin-destination pair (Pachon et al., 2006; Guerriero and Olivito, 2014);

* Transfer cost per unit of distance travelled in km (You and Hsieh, 2014);

* Fixed transfer cost (not dependent on the number of cars), depending on the origin-destination pair (You and Hsieh, 2014).

- Lost sales cost (You and Hsieh, 2014).

Profit / Value of rentals Most works that are operational-oriented (i.e., not developed under the revenue management framework) do not explain how the profit gained from each rental is pre-processed. Considering the business process, one assumes that the profit of a rental is a given parameter dependent on the origin, destination, starting date, length of rent, and car type requested (Oliveira et al., 2014). In some works, nevertheless, some simplifications are assumed, in accordance with the problem and other important assumptions. For example, You and Hsieh (2014) consider a given constant daily fee for all reservations, which is increased if the car is not returned to the same place where it was picked-up (note that in this work only one car type and one-day reservations are considered).

Uncertainty Some papers address deterministic versions of the problems in car rental fleet management (Conejero et al., 2014; Madden and Russell, 2012; Ernst et al., 2011; Hertz et al., 2009; Yang et al., 2009). All of those that consider uncertainty in the process, focus on demand for a specific product, which is thus the most relevant uncertain factor in these problems. Even in deterministic versions, for those papers that have a practical application, the given demand is said to be estimated based on historical data and forecasting techniques. Most works that consider demand to be uncertain state that it follows a certain distribution, such as Poisson (Haensel et al., 2012; Song and Earl, 2008) possibly altered by seasonality effects (You and Hsieh, 2014), Normal with different scenarios for its mean and variance (Guerriero and Olivito, 2014), discrete uniform (Li and Tao, 2010), or others (Steinhardt and Gönsch, 2012).

Moreover, there are other parts of the process subject to uncertainty. For example, Fink and Reiners (2006) claim that there is a significant level of uncertainty in the turnaround process (between rentals) that can be caused by delayed check-ins, need for repair, no-shows, among other factors. Nevertheless, they do not include this uncertainty in the model. Song and Earl (2008) consider uncertainty in the empty transfer times as well, modelling them with a probability distribution. 
Time span of decisions It is important to understand what time horizon is usually used for each type of problem. In fact, it will be shown that there are discrepancies between works that address the same problem and that there are overlaps between problems that are usually considered in separate strategic levels, on the modelling framework proposed by Pachon et al. (2006).

The pool segmentation main problem is to decide how to group rental locations into fleet-sharing pools. The reported time horizons for this decision were of 3 to 6 months (Yang et al., 2009). In fact, this decision is not necessarily rigid and can be updated more than once in a year to deal with changes in demand, among other factors.

Fleet size and mix is a decision that is taken monthly or each trimester (Pachon et al., 2006). However, decisions on acquisitions and removals, which logically impact fleet size, are reported to be taken in significantly different horizons, such as weekly (Fink and Reiners, 2006).

The fleet deployment within each pool is tackled in shorter time-spans, yet shows some discrepancies and sometimes overlaps with other decisions. It can be addressed daily (Pachon et al., 2006, 2003; You and Hsieh, 2014), weekly or every other week (Haensel et al., 2012), and considering a one-month horizon (Madden and Russell, 2012). If one considers the former, seminal works, these decisions are made in a five-day horizon (Geraghty and Johnson, 1997) and the decisions that influence size in a two-months (Geraghty and Johnson, 1997) to two-years (Carroll and Grimes, 1995) horizon.

The decisions on capacity allocation, namely whether or not to accept/reject requests, are taken considering one or two week horizons (Guerriero and Olivito, 2014; Steinhardt and Gönsch, 2012; Haensel et al., 2012). Pricing decisions are said to be made considering a one month horizon (Madden and Russell, 2012).

Even though a significant amount of works do not clearly define the time horizon considered, it is possible to conclude that most decisions not only share important links but can also be made in overlapping time horizons.

Other issues Other issues of the real-world setting of car rental fleet management problems are related with the behaviour of the consumer. No-shows - reservations made beforehand that are not fulfilled because the customer does not pick-up the vehicle - and cancellations - similar to no-shows, yet the customers notifies the company with some advance - are not usually considered. Walk-in customers - customers that arrive to a rental station and request a vehicle, without a previous reservation - are considered more often. Overbooking is a "typical" revenue management technique, yet, if applied, has significant operational implications and is usually not considered in vehicle-reservation assignment problems, in which it is critical. For example, Oliveira et al. (2014) consider that all reservations that were confirmed must be met and assume that there is always enough capacity to do so. Nevertheless, the importance of these issues is highly dependent on the problem considered.

As for each company's strategy to deal with lack of capacity, some works consider that there are no lost sales, i.e. all demand must be met, even if some capacity has to be sub-contracted at a significantly higher cost (Li and Tao, 2010; Hertz et al., 2009; Song and Earl, 2008).

As for more operational issues, usually maintenance constraints are only considered when tackling the assignment of reservations to specific vehicles (Oliveira et al., 2014; Hertz et al., 2009).

\section{Size of the problems}

Most of the works discussed in the last sections applied numerical examples to validate the results. Some of these were inspired or derived from real-world settings and problems faced by specific car rental companies. It is interesting to understand the size of the problems that was considered adequate to depict the reality of car rental companies, considering the different problems. Table 1 presents some of the main factors that influence the size of the problems for some of the works discussed before. The factors are characterized by the maximum values found in the instances of each paper. Not all factors that influence size are present but only the parameters derived from the real-world structure of the problem/business, which can help describe the different works in terms of their practical application.

The works that dealt with capacity allocation, i.e. accepting/rejecting booking requests, are not included in this table since the different approaches do not favour an unbiased comparison. Moreover, it is important to understand that the differences observed are often due to the level of complexity. For example, papers that dealt with fleet size/mix and deployment in an integrated manner show some discrepancies because of their significantly different goals: deriving general threshold policies versus developing models to solve real instances. Nevertheless, it was felt that this type of analysis could bring some insights, not as a comparison tool but as an overview tool for the assessment of the field. 
Table 1: Factors that influence the size of instances tested

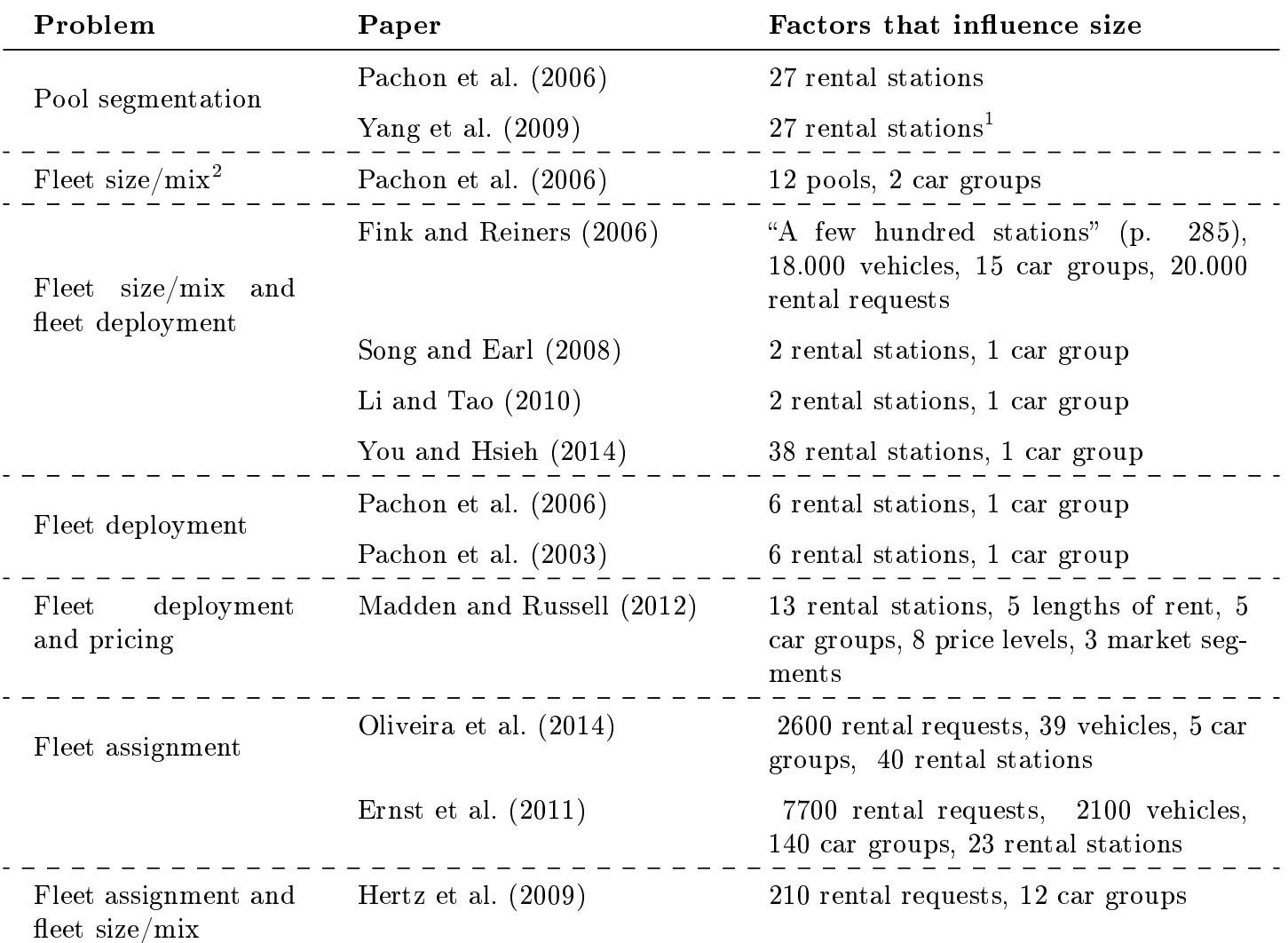

In fact, although some works already consider real-sized problems and instances, most of the assumptions made in this field still limit the applicability of the research in real-world settings. For example, it is interesting to notice that some works consider only one pool yet deal with more rental stations than the problems that aim to divide these into pools. Moreover, some assumptions and simplifications are arguably more realistic than others; fleet heterogeneity is said to be a critical characteristic of the car rental business and its inclusion on most problems significantly alters its structure, namely because of the possible upgrading strategies. In fact, the rental network design, especially the number of stations, and the characterisation of the fleet, especially the number of car groups, are generally felt to be the most important characteristics to increase realism and applicability. Nevertheless, for most problems, these are issues that have a significant impact on the size of problems and hence are often simplified.

\subsection{Methods}

Several quantitative methods have been applied in this field. Table 2 presents the methods and approaches followed by the works discussed in Section 3.1. The seminal works were excluded from this analysis since they mostly focus on decision support systems developed for specific companies, especially their structure and architecture, and the methods used are often not discussed in detail.

It is important to consider that, as it was explained in Sections 3.1 and 3.2, different assumptions and levels of "realism" were considered among the different papers. This has a significant influence in the choice of methodology, not only due to the complexity and dimensionality of the problems but also due to structural issues, such as nested upgrading strategies. Regarding the problems, it was decided to include only the general designation. However, some of the works tackling the same problem differed significantly on the assumptions made and issues considered. Regarding pool segmentation, the works of Yang et al. (2009) and Pachon et al. (2006) differ on the decisions, as the former work decides not only how the rental locations should be grouped in pools but also which location in each pool should be the pool logistics coordination center. As for fleet size, some works consider more detailed supply conditions on the acquisitions/removals issues, e.g. differentiating between leasing and buying (Hertz et al., 2009). It is

\footnotetext{
${ }^{1}$ Of these 27 stations, 11 are potential pool logistic centers.

${ }^{2}$ With integration of all pools in one problem
} 
important to mention that in this work the fleet size aspect is not the "core" decision: the increase of the size arises from situations of unavailability, due the requisite to fulfil all demand. However, other works consider simplified versions of the process (Pachon et al., 2006; Fink and Reiners, 2006) and others do not even consider this issue (You and Hsieh, 2014). Also regarding fleet assignment, as it was previously mentioned, Hertz et al. (2009) do not consider the possibility to reposition empty vehicles.

Table 2: Methods and approaches

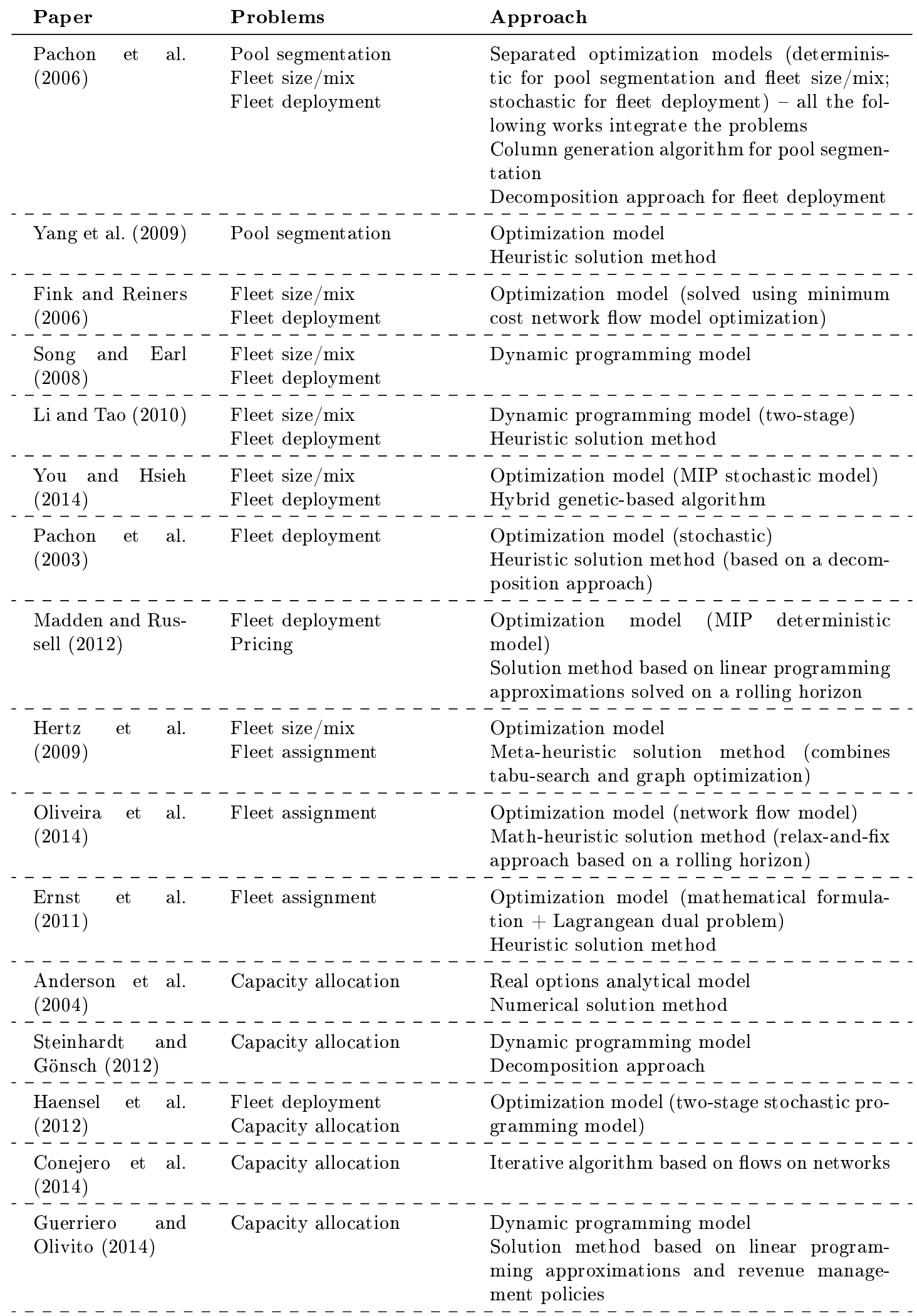


Continued from previous page

\begin{tabular}{|c|c|c|}
\hline Paper & Problems & Approach \\
\hline $\begin{array}{l}\text { Oliveira et al. } \\
(2015)\end{array}$ & Pricing & Adaptive heuristic procedure \\
\hline
\end{tabular}

It is possible to conclude that a large range of quantitative techniques has been used to address the different problems regarding car rental fleet management. It is also important to mention that in some works, such as Fink and Reiners (2006), simulation models were used as a tool to evaluate the robustness and quality of the solutions attained.

Although there is a tendency to use certain tools for specific problems (e.g. dynamic programming for capacity allocation), there is not a clear "methodological dominance". This seems reasonable in a field that is still growing and whose motivation arises from diverse areas, such as pricing or logistics, that traditionally recur to different methods.

It is also possible to observe that most works present a two-fold approach, where a model is presented and, due to its complexity or the dimensionality of the instances, a solution method to solve the model is proposed.

\section{A framework for the car rental fleet management problem}

\subsection{Proposed framework}

Building on the works of Pachon et al. (2006), a framework is herein proposed, with a different aim than the former and thus with a different perspective on the problem. Rather than providing a modelling framework, the goal was to structure the fleet management problem in the full car rental context, including revenue management and operational issues, in a more holistic yet detailed "map of action". That is to say, to consider, structure and frame the interactions between the usually isolated sub-problems studied by the companies, structuring a framework that is business-oriented rather than methodology-oriented.

The main goal of this framework is to open possibilities of research rather than to categorize the existing literature. Therefore, there is an attempt to follow the flow of the business decisions and to avoid restricting the decisions, e.g. based on geographical level or decision time horizon. The only "geographical" decision considered is the pool segmentation, since it is a building-block that can be easily disregarded if one chooses to consider the rental stations as a whole group and not divided in pools. Figure 2 represents the proposed framework.

The building blocks are connected by their main inputs/outputs, often in overlapping decision time horizons. Also, the key characteristic - flexibility - is present throughout the business process. In fact, most decisions are not "rigid" and can be frequently updated, from the most strategic ones, such as pool segmentation, to the most tactical ones, such as fleet assignment. That is represented in the framework with feedback loops in the processes where this is seen as critical. Moreover, the demand is a general input to the process, even though in different levels, and it should include not only unconstrained and constrained demand modelling/forecasting but also realistic issues such walk-in customers, no-shows and cancellations. The modelling of this core input is itself a research stream most relevant in this field.

As for the sources of the inputs/outputs of the building blocks, they can either be model decisions (inputs that are provided by other building blocks) or exogenous to these building blocks. Furthermore, the latter are divided in demand-related and non-demand-related inputs, due to the importance of demand modelling in this context.

As can be observed in Figure 2, the Pool segmentation building block receives as input exogenous information related with the network of rental stations of the car rental company, such as location and demand. The main decisions are related with the pool design. These will provide relevant information for all the subsequent building blocks, as they will focus on a single pool.

Fleet size/mix aims to decide how many vehicles of each group should comprise the fleet. As this involves a careful planning of the Acquisitions and removals - highly dependent on the time that the vehicles have been in the fleet - , it is significant to also decide on the best "vehicle age" mix. Besides "age"-related issues, other supply conditions must be considered within this block, such as supply modes (e.g. leasing), conditions (e.g. contract return date and/or "vehicle age") and costs (fixed or variable). To determine an adequate fleet size, it is also important to consider how the demand per vehicle group is distributed throughout the time horizon. The potential earnings are also critical inputs to decide on fleet size/mix. This information is highly dependent on the price setting and capacity allocation policies 

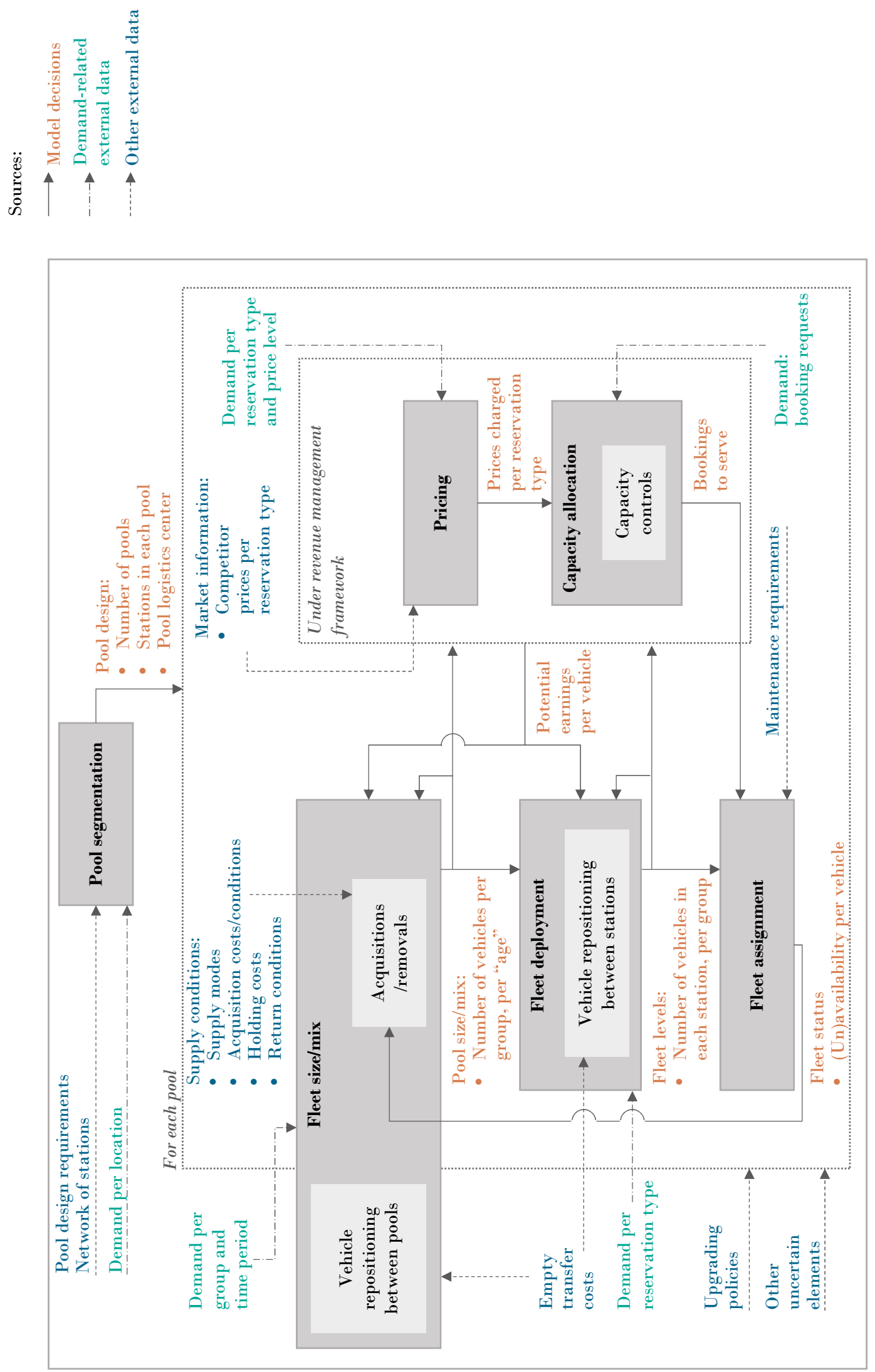

Figure 2: Framework for the car rental fleet management problem. The blocks represent different subproblems and the links between them the existing conceptual connections. Other relevant inputs are also represented. 
of the company. Moreover, if more than one pool is considered, it is important to consider that some vehicles may be currently in other pools (e.g. if the customer returned it to a different station) and it is necessary to plan the Vehicle repositioning between pools.

The main decisions of this block are the main inputs for Fleet deployment, which aims to determine how many vehicles of each group should be in each station. For this, the potential earnings are also essential inputs, as well as the empty transfer costs, since Vehicle repositioning between stations is a critical part of this problem. Here, the demand should be considered per reservation type. A "reservation type" encompasses reservations that require the same vehicle group and start and end in the same periods of time and in the same rental stations. This is important in order to understand not only the time distribution of demand but also its geographical distribution.

For Fleet assignment, it is important to know how the fleet is deployed between stations but also which reservations should be fulfilled by these vehicles, which is determined by the Capacity allocation. Maintenance constraints and requirements (especially dates of unavailability of specific vehicles) are also relevant exogenous information to this block. The main decision translates into a schedule of bookings to fulfil by each vehicle, which also provides useful information regarding unavailability when planning the fleet removals.

The problems that are usually tackled under the revenue management framework receive (and provide) significant inputs to the already mentioned building blocks, namely the number of available vehicles. Pricing is an issue that also requires external inputs such as market information regarding competitor prices, with a significant level of detail: usually, per reservation type. The high level of detail when encompassing demand inputs in this problem is also critical, as demand is not only dependent on the reservation type but is also highly sensitive to the price level. The Capacity allocation perspective on the revenue management car rental problem aims to select the bookings to serve within a list of booking requests (specific reservations made by customers for a certain price and with a certain antecedence), considering the limited availability of the fleet and defining the adequate Capacity controls, such as bid prices.

For all the building blocks of the framework that deal with problems within a specific pool, the upgrading policies are a relevant input, as it was previously discussed, since they connect the problems for different vehicle groups. Also, relevant uncertainties that affect the process, besides demand, should be considered (e.g. late reservation returns or unplanned maintenance).

From a complete and detailed view of this framework, together with an analysis of the existing literature, it is possible to identify potential research gaps and interests.

\subsection{Research gaps and directions}

\section{Research gaps}

Despite the fact that this is a growing field, where a significant body of research is still under development, it is interesting to understand what the most and the least studied problems are. Figure 3 presents a "heat map" of the field, based on the framework proposed on Section 4.1. The intensity of the greyscale increases proportionally with the amount of research, measured in number of papers ${ }^{3}$. Also, the connections between the different problems are analysed: the intensity of grey-scale is related to the number of papers that aim to integrate the connected problems. The papers considered are the ones presented in Table 2; therefore, it is important to notice that even the highest intensity refers only to eight papers.

The issue of fleet deployment is the most studied field, often in connection with the sizing issue. Nevertheless, the latter problem is not usually studied considering important characteristics of car rental fleet management: the inter-pool vehicle repositioning - due to the general assumption of "one pool perspective" when integrating with fleet deployment - and, especially, acquisitions and removals. As it was previously discussed, this is a significant aspect of the problem which is often oversimplified or even overlooked.

The pool segmentation problem has received little attention, as well as the fleet assignment; nevertheless, for the latter, the trend seems to be reverting in the past years. As for the former, this may be because the resulting pools are often confused with administrative divisions, which are much more strategic and difficult to update and adapt to demand and other external factors. Within the fleet deployment building-block, it is worth mentioning that the two main decisions - deciding fleet levels in each station

\footnotetext{
${ }^{3}$ Regarding the building-block "Acquisitions/Removals", included in "Fleet size/mix", only the papers where this issue was directly addressed (e.g. explicitly considering acquisition mode or removal location or date) were considered.
} 


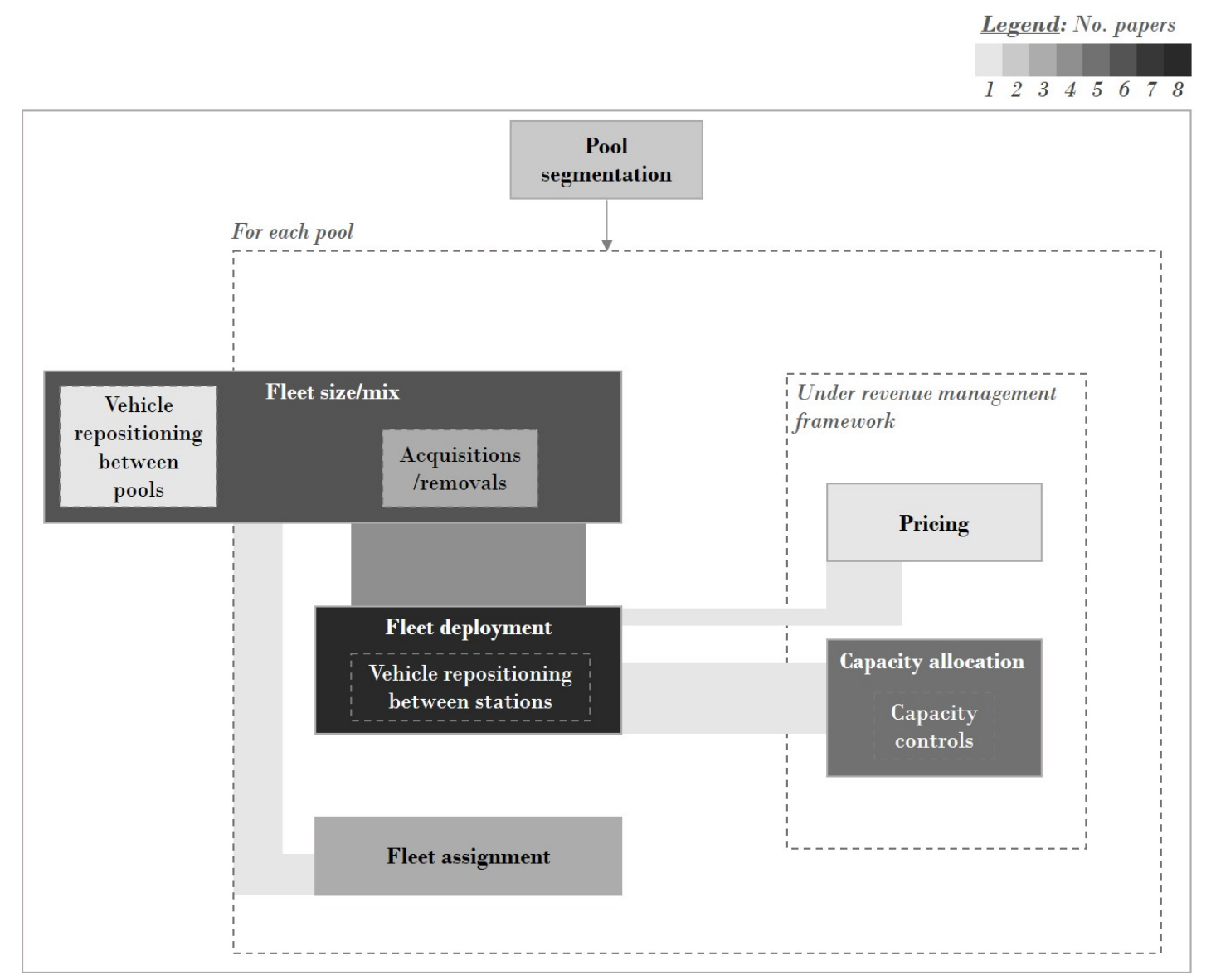

Figure 3: Heat map of the car rental fleet management literature. Based on the proposed framework, this heat map aims to show where most research has been focused and what areas are still developing. The grey intensity scale illustrates the number of papers focused on each problem and the integration of problems: from light grey (1 paper) to dark grey ( 8 papers)

and how to reposition vehicles among the stations - often lead to a decomposition in two sub-problems to facilitate the resolution (Pachon et al., 2006, 2003; You and Hsieh, 2014).

It is also interesting to notice that the relationship between operational problems and the ones within the revenue management framework, which are significantly dependent on operational decisions such as fleet size and deployment, namely capacity allocation, is still not very much developed. Also, as discussed before, pricing is a core issue for this sector and it is only addressed by one paper. Capacity allocation decisions, on the other hand, are the most studied problem within the revenue management framework.

\section{Research directions}

Firstly, it is important to establish that, as this is a problem that is still growing in the academic field, there are yet no saturated problems. Nevertheless, based on the review of the literature in the field, building on the proposed framework and keeping a close connection with the real-world applications in the car rental business, the following research directions are identified. Figure 4 "maps" these research directions to the framework presented in Figure 2, graphically referencing the sub-problems.

RD1) Increased realism to specific problems: due to the early stages of development of this field, there is the need to develop and extend the existing models, in order to encompass realistic assumptions, namely demand uncertainty, supply and resell relations, fleet heterogeneity and maintenance constraints;

RD2) Operational integration: the conceptual integration of the logistic/operational issues of fleet management (not studied under the revenue management framework) is mainly supported by the close interactions of the decisions, the discussion on their overlapping time horizons, and the inherent 


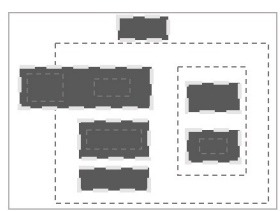

RD1) Increased realism

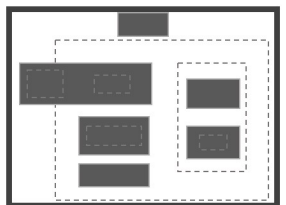

RD3) Overall

integration

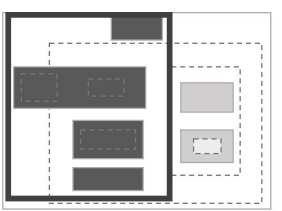

RD2) Operational integration

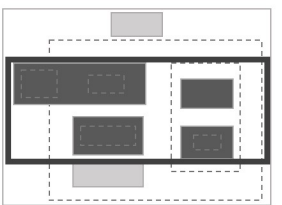

RD4) Horizontal integration

Figure 4: Research directions referenced to the proposed framework

flexibility of all the decisions considered, even if in different degrees. The conceptual integration of the problems does not necessarily mean that a "monolithic" solution approach should be used. In fact, the challenge of dimensionality will be present and innovative solutions to deal with it will be needed;

RD3) Overall integration: extending the integration proposed above, the encompassing integration of all building-blocks from the framework is supported by the reasons mentioned before and by the close links between the additional decision levels, discussed in Section 3.2. Despite the expected advantages of this full overview of the problem, the inherent challenge of dimensionality would be increased;

RD4) Horizontal middle-level integration: since the middle-level problems (fleet size/mix, deployment, assignment, pricing and capacity allocation) are the ones where the connections seem to be most critical and where the time horizons overlap the most, it would be interesting, especially in the perspective of a real-world application, to aim for an integration of these problems.

\section{Conclusions}

The main goal of this paper was to present, define and structure the car rental fleet management problem. In this process, three main contributions may be highlighted due to their relevance not only to the aforementioned goal but especially to support future developments in this field of research. Firstly, the topic was reviewed, encompassing a thorough discussion on the operational and revenue management issues of the problem. Secondly, based on the literature review, a framework was proposed to structure the car rental fleet management problem. Finally, based on this framework, four main research directions for the future are discussed. Overall, we believe the framework proposed, and its resulting contributions, will assist the field in its development where the focus on the business characteristics allied with a strong methodological background will allow the application in real-world settings of the research developed. Moreover, fleet management in some innovative transportation models, namely shared mobility systems, can also benefit from the contributions developed in this field, due to similarities found in the important differentiating characteristics: fleet and decision flexibility. Some interesting future work may therefore also lie in the expansion of this framework and resulting work to these systems.

\section{Acknowledgements}

The first author was supported by grant SFRH/BD/103362/2014 from Fundação para a Ciência e Tecnologia (Portugal). 


\section{References}

Anderson, C., M. Davison, and H. Rasmussen

2004. Revenue management: A real options approach. Naval Research Logistics, 51(5):686-703.

Anderson, C. K. and M. Blair

2004. Performance Monitor: The opportunity costs of revenue management. Journal of Revenue and Pricing Management, 2(4):353-367.

Auto Rental News

2015. U.S. Car Rental Revenue and Fleet Size Comparisons 2005-2015. [online] Retrieved from http://www.autorentalnews.com/fileviewer/2230.aspx [Acessed 14 June 2016].

Barth, M. and S. Shaheen

2002. Shared-Use Vehicle Systems: Framework for Classifying Carsharing, Station Cars, and Combined Approaches. Transportation Research Record, 1791(1):105-112.

Beaulieu, M. and M. Gamache

2006. An enumeration algorithm for solving the fleet management problem in underground mines. Computers and Operations Research, 33:1606-1624.

Bitran, G. and R. Caldentey

2003. An Overview of Pricing Models for Revenue Management. Manufacturing 6 Service Operations Management, 5(3):203-229.

Blair, M. and C. K. Anderson

2002. Performance Monitor. Journal of Revenue and Pricing Management, 1(1):57-66.

Carbajal, J. A., A. L. Erera, and M. Savelsbergh

2012. Balancing fleet size and repositioning costs in LTL trucking. Annals of Operations Research, 203(1):235-254.

Carroll, W. J. and R. C. Grimes

1995. Evolutionary Change in Product Management: Experiences in the Car Rental Industry. Interfaces, 25(5):84-104.

Clausen, J., A. Larsen, J. Larsen, and N. J. Rezanova

2010. Disruption management in the airline industry-Concepts, models and methods. Computers and Operations Research, 37(5):809-821.

Conejero, J. A., C. Jordán, and E. Sanabria-Codesal

2014. An iterative algorithm for the management of an electric car-rental service. Journal of Applied Mathematics, 2014.

Du, J. Y., J. O. Brunner, and R. Kolisch

2015. Obtaining the optimal fleet mix: A case study about towing tractors at airports. Omega, (in press).

Edelstein, M. and M. Melnyk

1977. The Pool Control System. Interfaces, 8:21-36.

Eftekhar, M., A. Masini, A. Robotis, and L. N. van Wassenhove

2014. Vehicle procurement policy for humanitarian development programs. Production and Operations Management, 23(6):951-964.

Ernst, A. T., E. O. Gavriliouk, and L. Marquez

2011. An efficient Lagrangean heuristic for rental vehicle scheduling. Computers $\&$ Operations Research, $38(1): 216-226$.

Feng, Y. and B. Xiao

2006. Integration of pricing and capacity allocation for perishable products. European Journal of Operational Research, 168(1):17-34. 
Fink, A. and T. Reiners

2006. Modeling and solving the short-term car rental logistics problem. Transportation Research Part E: Logistics and Transportation Review, 42(4):272-292.

Gallego, G. and G. Van Ryzin

1997. A multiproduct dynamic pricing problem and its applications to network yield management. Operations Research, 45(1):24-41.

Gamache, M., R. Grimard, and P. Cohen 2005. A shortest-path algorithm for solving the fleet management problem in underground mines. European Journal of Operational Research, 166:497-506.

Gavalas, D., C. Konstantopoulos, and G. Pantziou 2015. Design and Management of Vehicle Sharing Systems: A Survey of Algorithmic Approaches. In Smart Cities and Homes: Key Enabling Technologies, M. Obaidat and P. Nicopolitidis, eds. Elsevier Science.

Geraghty, M. K. and E. Johnson

1997. Revenue Management Saves National Car Rental. Interfaces, 27(1):107-128.

Gorman, M. F., K. Crook, and D. Sellers

2011. North American freight rail industry real-time optimized equipment distribution systems: State of the practice. Transportation Research Part C: Emerging Technologies, 19(1):103-114.

Guerriero, F., G. Miglionico, and F. Olivito

2012. Revenue management policies for the truck rental industry. Transportation Research Part E: Logistics and Transportation Review, 48(1):202-214.

Guerriero, F. and F. Olivito

2014. Revenue Models and Policies for the Car Rental Industry. Journal of Mathematical Modelling and Algorithms in Operations Research, 13(3):247-282.

Haensel, A., M. Mederer, and H. Schmidt

2012. Revenue management in the car rental industry: A stochastic programming approach. Journal of Revenue and Pricing Management, 11(1):99-108.

Hertz, A., D. Schindl, and N. Zufferey

2009. A solution method for a car fleet management problem with maintenance constraints. Journal of Heuristics, 15(5):425-450.

Lacetera, N., D. G. Pope, and J. Sydnor

2011. Heuristic Thinking and Limited Attention in the Car Market. National Bureau of Economic Research, No. w17030.

Li, Z. and F. Tao

2010. On determining optimal fleet size and vehicle transfer policy for a car rental company. Computers 63 Operations Research, 37(2):341-350.

Madden, T. and R. A. Russell

2012. Modelling the rental car pricing and relocation problem. International Journal of Revenue Management, 6(3-4):246-257.

Maglaras, C. and J. Meissner

2006. Dynamic pricing strategies for multiproduct revenue management problems. Manufacturing $\&$ Service Operations Management, 8(2):136-148.

Martinez, A. J. P., O. Stapleton, and L. N. van Wassenhove 2011. Field vehicle fleet management in humanitarian operations: A case-based approach. Journal of Operations Management, 29(5):404-421.

McAfee, P. R. and V. te Velde

2006. Dynamic pricing in the airline industry. In Handbook on Economics and Information Systems, T. Hendershott, ed., volume 1, Pp. 527-567. Elsevier. 
Netessine, S. and R. Shumsky

2002. Introduction to the Theory and Practice of Yield Management. INFORMS Transactions on Education, 3(1):34-44.

Oliveira, B. B., M. A. Carravilla, J. F. Oliveira, P. Raicar, D. Acácio, J. Ferreira, and P. Araújo 2015. Pricing for Internet Sales Channels in Car Rentals. In Operations Research and Big Data: IO2015XVII Congress of Portuguese Association of Operational Research (APDIO), A. Barbosa-Póvoa and J. L. de Miranda, eds., Pp. 139-147. Springer International Publishing.

Oliveira, B. B., M. A. Carravilla, J. F. Oliveira, and F. M. B. Toledo

2014. A relax-and-fix-based algorithm for the vehicle-reservation assignment problem in a car rental company. European Journal of Operational Research, 237(2):729-737.

Pachon, J., E. Iakovou, and C. Ip

2006. Vehicle fleet planning in the car rental industry. Journal of Revenue and Pricing Management, $5(3): 221-236$.

Pachon, J., E. Iakovou, C. Ip, and R. Aboudi

2003. A synthesis of tactical fleet planning models for the car rental industry. IEE Transactions, 35(9):907-916.

Pantuso, G., K. Fagerholt, and L. M. Hvattum

2014. A survey on maritime fleet size and mix problems. European Journal of Operational Research, $235(2): 341-349$.

Perrier, N., A. Langevin, and J. F. Campbell

2007. A survey of models and algorithms for winter road maintenance. Part IV: Vehicle routing and fleet sizing for plowing and snow disposal. Computers and Operations Research, 34:258-294.

Powell, W. B.

1991. Optimization Models and Algoritms: An emerging technology for the motor carrier industry. IEEE Transactions on Vehicular Technology, 40(1):68-80.

ReportsnReports

2015. Car Rental Market Growing at 15\% CAGR to 2019. [online] Retrieved from http://www.prnewswire.com/news-releases/car-rental-market-growing-at-15-cagr-to-2019510123371.html [Accessed 6 June 2016].

Salazar-González, J. J.

2014. Approaches to solve the fleet-assignment, aircraft-routing, crew-pairing and crew-rostering problems of a regional carrier. Omega, 43:71-82.

Sayarshad, H. R., N. Javadian, R. Tavakkoli-Moghaddam, and N. Forghani 2010. Solving multi-objective optimization formulation for fleet planning in a railway industry. Annals of Operations Research, 181:185-197.

Şen, A.

2013. A comparison of fixed and dynamic pricing policies in revenue management. Omega, 41:586-597.

Shaheen, S. A.

2013. Introduction Shared-Use Vehicle Services for Sustainable Transportation: Carsharing, Bikesharing, and Personal Vehicle Sharing across the Globe. International Journal of Sustainable Transportation, $7(1): 1-4$.

Sherali, H. D., E. K. Bish, and X. Zhu

2006. Airline fleet assignment concepts, models, and algorithms. European Journal of Operational Research, 172:1-30.

Sherali, H. D. and A. B. Suharko

1998. A tactical decision support system for empty railcar management. Transportation Science, $32(4): 306-329$.

Song, D.-P. and C. F. Earl

2008. Optimal empty vehicle repositioning and fleet-sizing for two-depot service systems. European Journal of Operational Research, 185(2):760-777. 
Spieckermann, S. and S. Voß

1995. A case study in empty railcar distribution. European Journal of Operational Research, 87(95):586598.

Steinhardt, C. and J. Gönsch

2012. Integrated revenue management approaches for capacity control with planned upgrades. European Journal of Operational Research, 223(2):380-391.

Talluri, K. T. and G. Van Ryzin

2006. The Economics of RM. In The Theory and Practice of Revenue Management, chapter 8, Pp. 333405. Springer Science \& Business Media.

Van Ryzin, G. J. and K. T. Talluri

2005. An Introduction to Revenue Management. In Tutorials in Operations Research, Pp. 142-194.

Weatherford, L. R.

1997. Using prices more realistically as decision variables in perishable-asset revenue management problems. Journal of Combinatorial Optimization, 1:277-304.

Yang, Y., W. Jin, and X. Hao 2008. Car rental logistics problem: a review of literature. IEEE International Conference on Service Operations and Logistics, and Informatics. IEEE/SOLI 2008, 2:2815-2819.

Yang, Y., W. Jin, and X. Hao

2009. Dynamic Pool Segmentation Model and Algorithm in the Car Rental Industry. Journal of Computers, 4(12):1202-1208.

You, P.-S. and Y.-C. Hsieh

2014. A study on the vehicle size and transfer policy for car rental problems. Transportation Research Part E: Logistics and Transportation Review, 64:110-121.

Żak, J., A. Redmer, and P. Sawicki

2011. Multiple objective optimization of the fleet sizing problem for road freight transportation. Journal of Advanced Transportation, 45:321-347. 